\title{
Path integral study of the role of correlation in exchange coupling of spins in double quantum dots and optical lattices
}

\author{
Lei Zhang, ${ }^{1}$ M. J. Gilbert, ${ }^{2,3}$ Jesper Goor Pedersen, ${ }^{4}$ and J. Shumway ${ }^{1, *}$ \\ ${ }^{1}$ Deptartment of Physics, Arizona State University, Tempe, AZ 8528\%-1504, USA \\ ${ }^{2}$ Microelectronics Research Center, University of Texas, Austin, Texas 78758, USA \\ ${ }^{3}$ Department of Electrical and Computer Engineering, University of Illinois, Urbana, IL 61801, USA \\ 4 DTU Fotonik, Department of Photonics Engineering, \\ Technical University of Denmark, Kongens Lyngby, Denmark
}

(Dated: October 22, 2018)

\begin{abstract}
We explore exchange coupling of a pair of spins in a double dot and in an optical lattice. Our algorithm uses the frequency of exchanges in a bosonic path integral, evaluated with Monte Carlo. This algorithm is simple enough to be a "black box" calculator, yet gives insights into the role of correlation through two-particle probability densities, visualization of instantons, and pair correlation functions. We map the problem to Hubbard model and see that exchange and correlation renormalize the effective parameters, dramatically lowering $U_{r}$ at larger separations.
\end{abstract}

PACS numbers: 73.21.La, 37.10.Jk, 75.30.Et, 02.70.Ss

Lattice models are popular in solid state physics, often serving as simple models for atomic orbitals, especially in the theory of magnetism [1]. Quantum dot (QD) arrays and optical lattices (OL) have emerged as new realizations of lattices. These artificial lattices are candidates for quantum computers, where spins on exchangecoupled QDs act as qubits for universal quantum computation [2, 3]. A basic concept of lattice models is the intersite exchange, in which virtual hopping leads to an effective spin coupling of neighboring sites. A two-site model is one of the simplest quantum problems, yet the quantitative mapping from a three-dimensional model of a recent double QD or OL experiment to an effective two-site Hamiltonian has many subtleties requiring careful treatment of exchange and correlation $[1,3,4]$.

In this Letter we develop a simple path integral Monte Carlo (PIMC) approach for extracting accurate singlettriplet splitting from a two- or three-dimensional continuous model. This two-particle problem has been previously solved with direct diagonalization (DD) methods with a careful choice of basis functions [4] and is amenable to variational or diffusion quantum Monte Carlo (QMC) [5]. However, the simple and elegant PIMC approach is a more direct solution without variational bias or basisset issues and offers theoretical insights into this important problem. We first show that the splitting energy, $J$, is easily extracted from the average permutation of the two-particle path integral, even when $J \ll k_{B} T$. This PIMC algorithm can be a black-box calculator, providing accurate numerical estimates of $J$ for technologically relevant models of quantum dots or optical lattices, with arbitrary interactions and confinement potentials. The path integral also allows us to ask questions about the correlated behaviour of the electrons. For example, do the electrons exchange across the barrier simultaneously, or do they briefly double occupy the dot? Or, how does the motion of one electron over the barrier correlate with the location of the other electron? We answer these questions by viewing representative trajectories (instantons) in the path integral, and, more quantitatively, by calculating pair correlation functions. Next, we include the effects of a magnetic field on electron exchange in double QDs using a Berry's phase for the magnetic flux enclosed by the electron paths [6]. Finally we use the method to model recent experiments of exchange coupled atoms in an optical trap, demonstrating its broad utility [7].

The mapping from a continuous model with interacting particles to a lattice model introduces subtle complications. For a non-interacting system it is reasonable to reduce the Hilbert space to include just one orbital per site, such as a Wannier function centered on each potential minimum. The non-interacting many-body ground state is a product state of these single particle orbitals, and low-lying excited states are also spanned by this basis, so an effective lattice model is an excellent approximation. Interactions are typically added to this lattice model, often as on-site energies, $U$, or intersite terms, $V$. For small $t$, this gives the well-known $J=-4 t^{2} /(U-V)$.

There can be a serious flaw when considering interactions in this order, by first mapping to a lattice then adding interactions. When interactions are added to the continuum Hamiltonian, correlation enters as virtual excitations to higher energy orbitals. At first this seems insignificant, since there may be still a one-to-one mapping to an effective lattice model. But, when choosing effective lattice parameters, one must remember that many-body states in the continuum model have quantum fluctuations that are simply not present in the lattice model.

As a specific example, consider two electrons in two coupled QDs. This system is often represented at a twosite Hubbard model, where the sites represent the $1 \mathrm{~s}$ ground states of the quantum dots. Correlation terms involve virtual excitation of the electron to the $2 p_{x}$. and $2 p_{y}$ states of the dots. These quantum fluctuations give 
rise to van der Waals attraction, in addition to the usual mean-field Coulomb repulsion. Van der Waals attraction and other correlations renormalize the interaction parameters to new values, $U_{r}$ and $V_{r}$.

When we consider hopping between sites, more complications emerge. The hopping barrier has contributions from both the external potential and electron-electron interactions. While the mean-field Hartree contribution can simply be added to the effective potential, the fluctuating part is not so trivial. In the transition state, an electron passes over a barrier whose height has quantum fluctuations. Thus we expect interactions to renormalize the hopping constant, $t_{r}$. At the Hartree-Fock level, Hund-Mulliken theory already predicts a renormalized $t_{r}$ and $U_{r}$ due to long-range exchange. However, the neglect of correlation in Hund-Mulliken theory can lead to catastrophic failure at intermediate dot separations [4]. Our PIMC approach includes all correlation and illuminates its role in barrier hopping with the concept of instantons.

We start with the two-dimensional model for the GaAs double quantum dot studied in Ref. [4],

$$
H=\frac{\mathbf{p}_{1}^{2}}{2 m^{*}}+\frac{\mathbf{p}_{2}^{2}}{2 m^{*}}+\frac{e^{2}}{\epsilon\left|\mathbf{r}_{1}-\mathbf{r}_{2}\right|}+V_{\text {ext }}\left(\mathbf{r}_{1}\right)+V_{\text {ext }}\left(\mathbf{r}_{2}\right),
$$

with $m^{*}=0.067 m_{e}$ and $\epsilon=12.9$. The external potential comes from two piecewise-connected parabolic potentials,

$$
V_{\text {ext }}(\mathbf{r})=\frac{1}{2} m \omega_{0}\left\{\min \left[(x-d)^{2},(x+d)^{2}\right]+y^{2}\right\},
$$

with minima at $x= \pm d$. We report $d$ relative to the oscillator length $r_{0}=\sqrt{\hbar / m \omega_{0}}$. The two lowest energy two-electron states are spatially symmetric and antisymmetric under exchange, with energies $\varepsilon_{+}$and $\varepsilon_{-}$, respectively. The exchange coupling, $J=\varepsilon_{-}-\varepsilon_{+}$, has been calculated previously using DD on a basis of Fock states built from seven single particles states [4]. Much care was taken to test convergence with the number of states and careful evaluation of coulomb matrix elements. We note that the same quality of DD calculation in three dimensions would typically take more single particle states.

QMC techniques give essentially exact answers to many problems without basis set convergence issues, and often work just as easily in two or three dimensions. PIMC is nice for QD problems [8] because it does not require a trial wavefunction. However, a direct calculation of either $\varepsilon_{+}$or $\varepsilon_{-}$with PIMC often has a large statistical errors in energy $(\sim 1 \mathrm{meV}$ in QDs). Instead, we use particle exchange statistics to estimate energy difference $J$ to high accuracy $(\sim 1 \mu \mathrm{eV})$ in PIMC.

To calculate $\mathrm{J}$, we split the partition function into two parts that are either spatially symmetric and antisymmetric under exchange, $Z=Z_{+}+Z_{-}$. These two terms can be expressed as a symmetrized and antisymmetrized imaginary-time path integral, [9, 10]

$$
Z_{ \pm}=\frac{1}{2 !} \sum_{P=\mathcal{I}, \mathcal{P}}( \pm 1)^{P} \int \mathcal{D} R(\tau) e^{-\frac{1}{\hbar} S_{E}[R(\tau)]}
$$

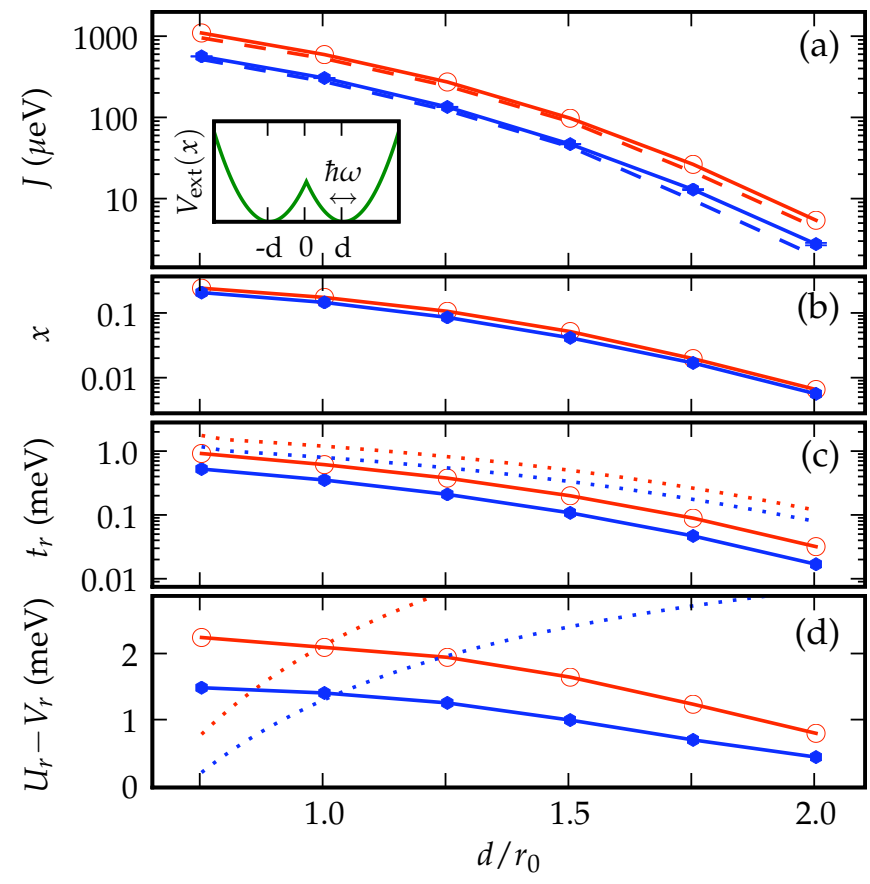

FIG. 1: (Color online) PIMC results. (a) Exchange couplings $J$ for $\hbar \omega=4 \mathrm{meV}(\bullet)$ and $\hbar \omega=6 \mathrm{meV}$ (o) double QDs with a piecewise parabolic potential (inset). Dashed lines are direct diagonalization results from Ref. 4. (b) The double dot occupation probability $x$. Using $J$ and $x$ we fit (c) $t_{r}$ and (d) $U_{r}$ parameters for an effective two site Hubbard-Model. Dashed lines in (c) show the bare hopping $t$ for a single electron in the double QD, while the dashed line (d) is for $V=e^{2} / \epsilon d$ and $U$ taken from a PIMC calculation on a single QD.

This is a sum over all two-particle worldlines $R(\tau)$ with the boundary condition $R(\beta \hbar)=P R(0)$, where the operator $\mathcal{P}$ swaps the particles. The symbol $( \pm 1)^{P}$ takes on the values $( \pm 1)^{\mathcal{I}}=1,(+1)^{\mathcal{P}}=1$, and $(-1)^{\mathcal{P}}=-1$. At low temperature, only the two lowest states contribute to the partition functions, so $Z_{ \pm}=e^{-\beta \varepsilon_{ \pm}}$. Then,

$$
e^{-\beta J}=\frac{Z_{-}}{Z_{+}}=\frac{\sum_{P} \int \mathcal{D} R(\tau)(-1)^{P} e^{-\frac{S_{E}}{\hbar}}}{\sum_{P} \int \mathcal{D} R(\tau) e^{-\frac{S_{F}}{\hbar}}} \equiv\left\langle(-1)^{P}\right\rangle_{+} .
$$

or $J=-k_{B} T \ln \left\langle(-1)^{P}\right\rangle_{+}$. Thus the exchange coupling can be calculated by sampling a symmetric (bosonic) path integral [10] and taking the average of $(-1)^{P}$, which is +1 for identity paths and -1 for exchanging paths.

We ran PIMC simulations [10] with our open-source pi code for the dots studied in Ref.4, with the results shown in Fig. 1 (a). While each geometry was sampled for four hours on eight cores, the algorithm can be ran for just one minute to get quick answers with larger error bars. To aid other researchers, we have made the simulation available as a tool on nanoHUB [11]. Coulomb interactions are included with a crude pair action that correctly handles the cusp condition. We observed convergence of the path integral results with 6400 discrete slices, but a higher- 
(a)

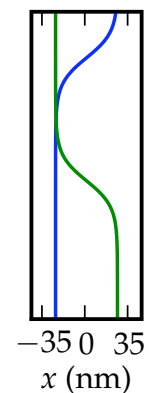

(d)

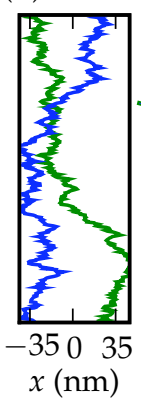

(b)

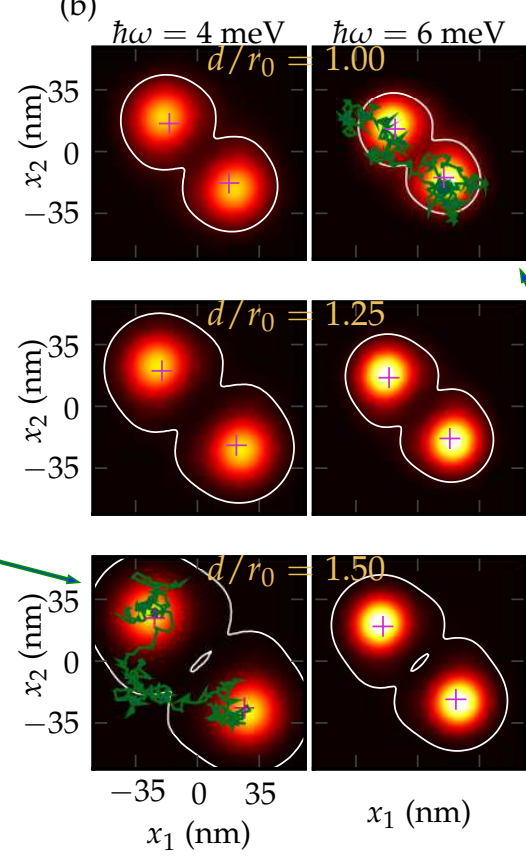

(c)

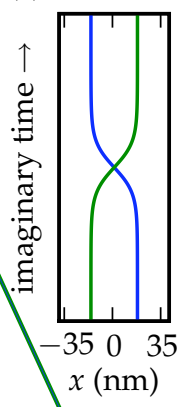

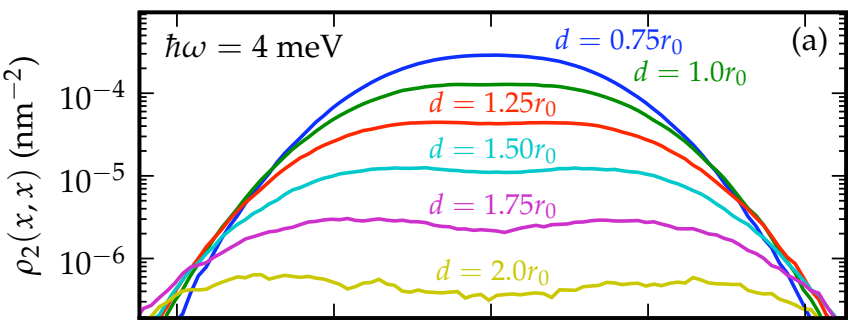

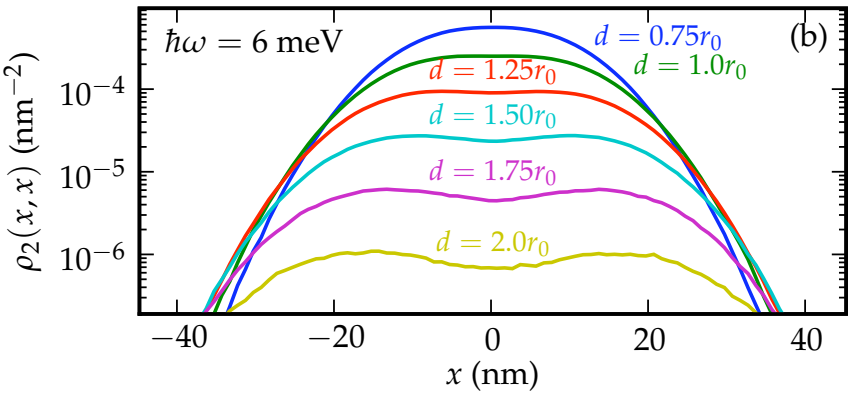

FIG. 3: (Color online) Crossing density, $\rho_{2}(x, x)$, equivalent to the diagonal of the pair densities in Fig. 2(b).

occupation of a dot, illustrated in Fig. 2(a), or simultaneous exchange, as in Fig. 2(c). In Fig. 2(d) and (e) we show actual paths sampled from our simulation that resemble the idealized instantons. In Fig. 2(c), one instanton can be seen to move from the $(d,-d)$ minimum, briefly double-occupy the left dot, $(-d,-d)$, then move to the $(-d, d)$ minimum, while the other instanton moves directly between the two minima.

Contours of $\rho\left(x_{1}, x_{2}\right)$, Fig. 2(b), illustrate a trend with increasing dot separation. For small $d$ the highest probability is directly between the minima (simultaneous exchange), but at larger $d$ the highest probability has two pathways (brief double occupation). Fig. 3 show the probability density for crossing, $\rho(x, x)$. Crossing is most likely in the middle $(x=0)$ when the dots are close together. When the dots are farther apart, the crossing probability has a double peak near $x= \pm d$, while the probability for crossing in the middle is approximately half the peak value. The double peaks are slightly larger for the wider $\hbar \omega=4 \mathrm{meV}$ dot, consistent with more double occupation, but the difference is not very pronounced.

To underscore the presence of electronic correlation during tunneling, we plot the correlation hole form in Fig. 4, with PIMC results next to DD results 4]. While some quantitative differences are apparent, consistent with the finite basis size in DD, the overall agreement is quite good. The message is clear: in the instanton, as one electron moves between the dots, the other electron moves away, enhancing the instanton and increasing $J$.

For charged particles, magnetic fields can be used to tune the exchange coupling and even change its sign [3]. In the path integral, a magnetic field is easily implemented as a Berry's phase $q \Phi_{B}$, where $q$ is the electron charge and $\Phi_{B}$ is the total magnetic flux enclosed by the

ings called instantons, in which particles exchange tween the dots. An instanton can involve brief double- 


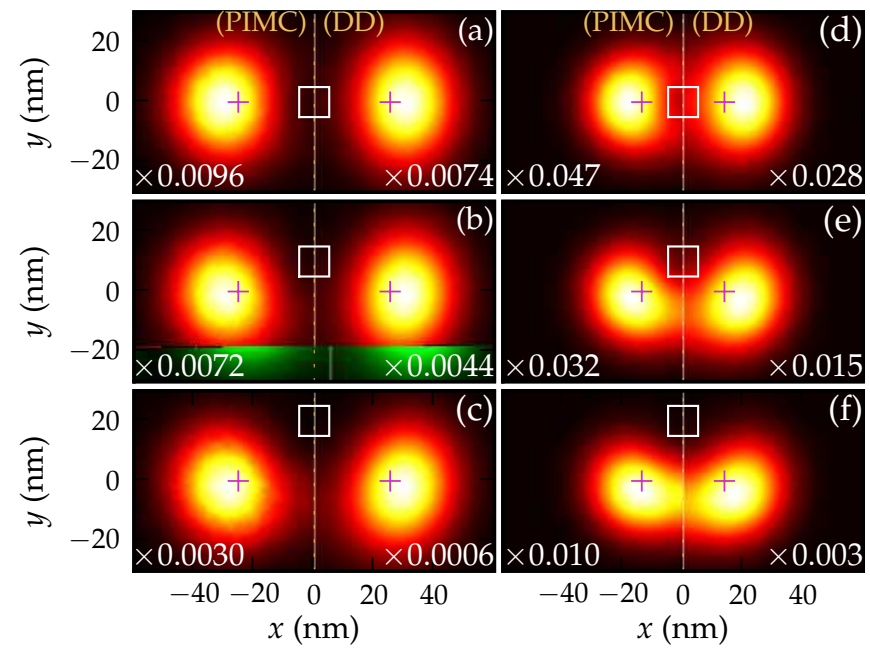

FIG. 4: (Color online) Conditional density of one electron when the other electron is in the white box, showing the correlation hole during an instanton. Panels (a)-(c) are the $\hbar \omega=4 \mathrm{meV}, d=1.5 r_{0}$ QDs and (d)-(f) are the $\hbar \omega=6 \mathrm{meV}$, $d=1.0 r_{0}$ QDs. Numerical factors are the likelyhood of the first electron being in the white box. PIMC results are shown on the left of each image, with DD results [4] on the right.

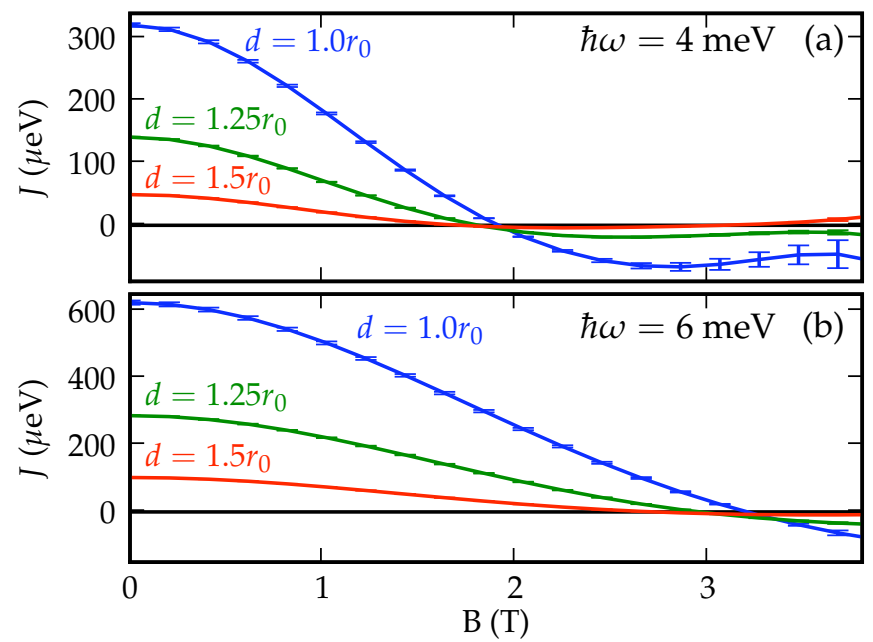

FIG. 5: (Color online) Magnetic field dependence included with a Berry's phase for several double QDs.

worldlines of the two electrons. The exchange splitting is then $J(B)=-k_{B} T \ln \left(\left\langle e^{i q \Phi_{B}}(-1)^{P}\right\rangle_{+} /\left\langle e^{i q \Phi_{B}}\right\rangle_{+}\right)$. The quantities are averaged from the bosonic path integral with no field, so data for different B-fields may be collected simultaneously. For very large magnetic fields the expectation value in the denominator is small and Monte Carlo sampling errors are catastrophic. In practice, we find that fields up to $4 \mathrm{~T}$ in strength are practical for the geometries we study, yielding the results in Fig. 5 .

As a final example, consider the exchange of two ${ }^{87} \mathrm{Rb}$ atoms in a double-well optical trap [7]. This system resembles the double QD, only with much heavier particles, a much lower temperature, short range interactions,

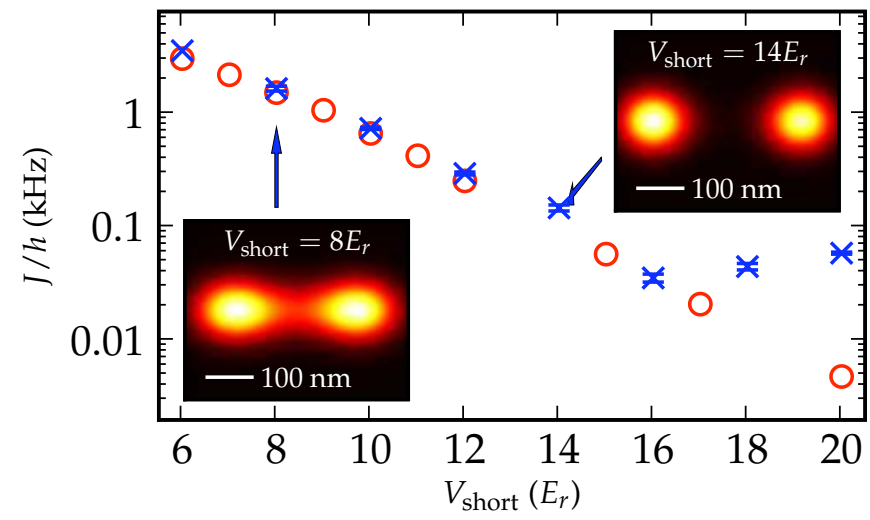

FIG. 6: (Color online) Spin-splitting of ${ }^{87} \mathrm{Rb}$ atoms trapped in a double well: $\times$, PIMC results at $10 \mathrm{nK}$, and $\circ$, experimental data [7]. Insets show atomic probability densities.

and a different confining potential. While the experiments in [7] have very little correlation, we present results here to show the method is practical for such systems and could be used for predictions of strongly interacting systems. The experiment has a double-well potential, $V(x)=V_{\text {long }} \sin ^{2}(\pi x / \lambda)+V_{\text {short }} \cos ^{2}(2 \pi x / \lambda)$, with $\lambda=765 \mathrm{~nm}$ and $V_{\text {long }}=10 E_{r}$, where $E_{r}=h^{2} / 2 M_{\mathrm{Rb}} \lambda^{2}$ 7]. We model interactions as $V(r)=V_{0} \operatorname{sech}^{2} \kappa r$ with $V_{0}=50.5 \mu \mathrm{K}$ and $\kappa=0.1 \mathrm{~nm}^{-1}$ to reproduce the ${ }^{87} \mathrm{Rb}$ scattering length. Fig 6 shows $J$ as the barrier $V_{\text {short }}$ is varied, confirming agreement with experiment.

In conclusion, we have demonstrated a PIMC algorithm for computing exchange-splitting in double QDs and optical lattices. The exchange splitting arises from instantons in the path integral, and we have collected statistics for these path crossings, including double occupation and the correlation hole. Correlations renormalize $t_{r}$ and $U_{r}-V_{r}$, with dramatic decrease in $U_{r}-V-r$ at large separation. We find that simultaneous crossing occurs more often with closely spaced dots, while further separated dots are more likely to have instantons with double occupations. Finally, we have demonstrated the versatility of the algorithm with the inclusion of magnetic fields and applications to laser-trapped atoms.

Work supported by NSF Grant No. DMR 0239819 and NRI-SWAN and made use of facilities provided by the Ira A. Fulton High Performance Computing Initiative. JS thanks Erich Mueller for helpful discussions.

* shumway@mailaps.org http://phy.asu.edu/shumway

[1] D. C. Mattis, The Theory of Magnetism, no. 17 in Springer series in solid-state science (Springer-Verlag, Berlin, 1981).

[2] D. Loss and D. P. DiVincenzo, Phys. Rev. A 57, 120 (1998).

[3] G. Burkard, D. Loss, and D. P. DiVincenzo, Phys. Rev. 
B 59, 2070 (1999).

[4] J. Pedersen, C. Flindt, N. A. Mortensen, and A.-P. Jauho, Phys. Rev. B 76, 125323 (2007).

[5] A. Ghosal, A. D. Güçlü, C. J. Umrigar, D. Ullmo, and H. U. Baranger, Nature Physics 2, 336 (2006).

[6] M. V. Berry, Proc. R. Soc. A 392, 45 (1984).

[7] S. Trotzky, P. Cheinet, S. Folling, M. Feld, U. Schnorrberger, A. M. Rey, A. Polkovnikov, E. A. Demler, M. D. Lukin, and I. Bloch, Science 319, 295 (2008).
[8] M. Harowitz, D. Shin, and J. Shumway, J. Low Temp. Phys. 140, 211 (2005).

[9] R. P. Feynman, Statistical Mechanics (Addison-Wesley, Reading, MA, 1972).

[10] D. M. Ceperley, Rev. Mod. Phys. 67, 279 (1995).

[11] J. Shumway and M. Gilbert, Spin coupled quantum dots (2008), doi: 10254/nanohub-r4963.1. 